\title{
Simulated satellite imagery of lengthy territories with complex configuration
}

\author{
Dmitriy Mozgovoy ${ }^{1, *}$, Dmitriy Svinarenko ${ }^{1}$, Roman Tsarev $^{2}$, and Tatiana Yamskikh $^{2}$ \\ ${ }^{1}$ Oles Honchar Dnipropetrovsk National University, 72, Gagarin Prospect, Dnipropetrovsk, 49000, \\ Ukraine \\ ${ }^{2}$ Siberian Federal University, 79, Svobodny Prospect, Krasnoyarsk, 660041, Russia
}

\begin{abstract}
The study presents the results of creating simulated satellite imagery of lengthy territories with complex configuration using data from satellites with optical-electronic scanners of ultrahigh spatial resolution. The article attempts to clarify the feasibility of one-orbit imagery of a given lengthy object, taking into account the existing limitations on the values of pointing angles and satellite angular velocities based on the results of modeling the system of automatic satellite attitude program control during the process of imagery.
\end{abstract}

\section{Problem statement}

When solving a number of economic and scientific problems, there arose a need for fast satellite imagery of lengthy territories with complex configuration (highways and railroads, oil and gas pipelines, border areas, navigable waterways, coastlines, etc.) [1-6]. For this purpose ultrahigh resolution remote sensing satellites are commonly used. The distinctive features of Earth remote sensing satellites with optical-electronic scanners providing ultrahigh spatial resolution are as follows [1, 7-10]:

1) a narrow swath width, which does not allow to take images of arbitrarily-spaced lengthy territories in one-orbit period;

2) a small pixel size of CCD photocells (i.e., low sensitivity), which does not allow to make images of low-contrast objects, as well as take pictures from neighboring orbits at low angles of the Sun.

To cover a lengthy object with an arbitrary configuration, it is necessary to take several images from different orbits, which takes from 3 days to 3 weeks. It may take even longer time due to cloudiness, which is unacceptable for most tasks.

\section{Possible solutions}

In the remote sensing satellites with optical-electronic scanners of ultra-high spatial resolution existing abroad, the following problems have been partially solved:

\footnotetext{
* Corresponding author: $\mathrm{m}-\mathrm{d}-\mathrm{k} @$ i.ua
} 
- some satellites allow to take images of arbitrarily oriented straight-line lengthy territories (Fig. 1, in the middle), choosing the most optimal scanning direction (however, imagery of a lengthy territory with complex configuration is still performed in several orbits);

- for some satellites it is possible to take images increasing the exposure time (i.e., take pictures of low contrast or low light objects), but in this case the scene has a shape of a rectangle.
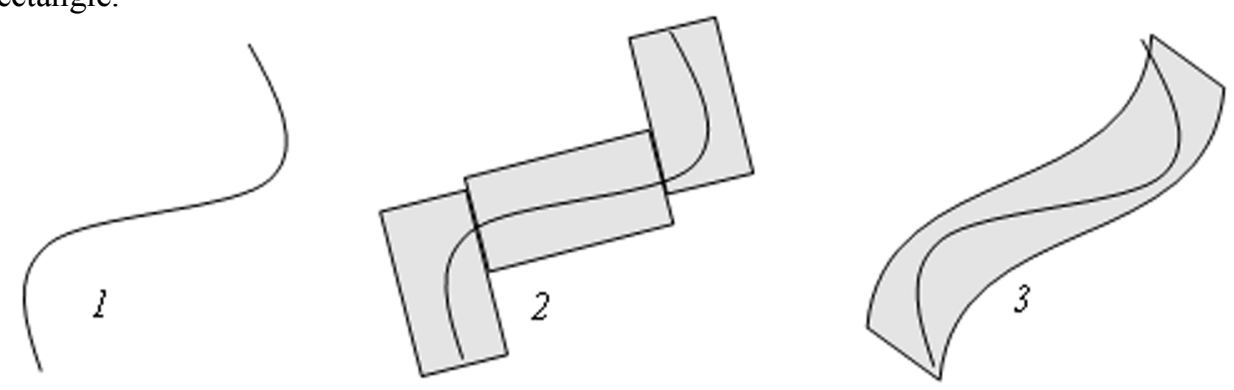

Fig. 1. Options for satellite imagery of the lengthy object

(1 - Target; 2 - Three-orbit imagery; 3 - One-orbit imagery)

\section{The nature of a new method of satellite imagery}

An advanced technique implemented using automatic satellite attitude program control in the process of retargeting (i.e., with non-zero angular velocities) may significantly increase the efficiency of satellite imagery of lengthy territories with complex configuration.

In this case, it is possible to choose the best scan mode to take images of arbitrarilyspaced lengthy territories in one-orbit period, i.e. much faster (Fig. 1, on the right). Moreover, non-zero angular velocities allow to increase the exposure time and thus improve image classification procedures using radiometric and spectral characteristics.

\section{Limitations of the proposed method}

The use of automatic satellite attitude program control system of satellite angular motion imposes increased requirements on the on-board attitude system both in terms of providing the required attitude accuracy and stabilization of the satellite, and in providing the required retargeting velocity directly during the imagery. Moreover, special requirements apply to the subsystem of magnetic discharge of inertial energy-storage devices (flywheels).

Given these limitations, caused by a more intensive retargeting of the satellite directly in the process of imagery, and, as a result, increased power consumption, there arose a necessity for computer modeling of new satellite imaging modes with ultra-high spatial resolution scanners.

\section{The main stages of research}

The main stages of modeling satellite imagery of lengthy territories with complex configuration:

- approximation of a lengthy object defined by separate nodal points on a digital map (linear, quadratic, spline, etc.); 
- determination of the optimal coverage of a lengthy object, taking into account the swath width of the imaging instrument and the required scanning direction;

- calculation of the orbital motion in order to choose the orbit for imagery and the time for switching on the imaging instrument, taking into account the Delay coefficient (when implementing the "Time Delay and Integration" mode);

- calculation of the satellite attitude angles required for imaging a given lengthy object, taking into account the installation angles of the imaging instrument;

- estimation of the satellite angular velocities during the imagery and analysis of the imagery feasibility, taking into account the existing limitations on the values of pointing angles and satellite angular velocities;

- modeling of satellite imagery process, taking into account additional factors (atmospheric refraction, terrain relief displacement, etc.);

- estimation of the impact of systematic and random errors on the accuracy of obtaining the object coordinates from the image (errors in the installation of the imaging instrument, errors in determining the satellite attitude and position parameters, etc.).

\section{The description of mathematical models used}

\subsection{Mathematical model of a lengthy territory with complex configuration}

The territory with complex configuration to be imaged is marked on the map by nodal points with arbitrary step in latitude and longitude. To approximate the lengthy territory, a natural cubic interpolating spline is used, then smoothed with the least squares method.

\subsection{Determination of satellite attitude in the process of imagery}

The input data are composed of:

- coordinates of the reference points in geographic coordinate system corresponding to start and end points of the line;

- satellite coordinates in geographic coordinate system;

- pointing vectors of the reference points in the instrument coordinate system;

- the values of the elements in the transition matrix from the orbital coordinate system to the geographic coordinate system.

Yaw, pitch and roll attitude angles and angular velocities identify a reference frame that moves with the satellite.

The calculation procedure is as follows:

- normalised vectors of the reference points alignment in geographic coordinate system are calculated;

- the values of the elements in the transition matrix from the geographic coordinate system to the orbital coordinate system are determined;

- vectors of the reference points alignment in the orbital coordinate system are calculated;

- the values of the elements in the transition matrix from the instrument coordinate system to the orbital coordinate system are defined;

- yaw, pitch and roll attitude angles are determined by the values of the elements in the transition matrix from the instrument coordinate system to the orbital coordinate system.

Angular velocities are found by analytical differentiating the coefficients of the spline interpolating the attitude angles. 


\subsection{The model of satellite orbital motion}

For short-term prediction and low earth circular orbits, sufficient accuracy is achieved with the propagator SGP4, which allows to work with the initial conditions in TLE files generated by NORAD.

\section{The input data for modeling}

The input data for modeling satellite imagery of lengthy territories with complex configuration are as follows:

- the territory to be imaged is $600 \mathrm{~km}$ in length;

- the type of satellite orbit - solar-synchronous, with about $700 \mathrm{~km}$ height;

- the scanner swath width at nadir $-20 \mathrm{~km}$.

The following types of imagery were modeled:

- without implementation of TDI (Time Delay and Integration) mode (spline approximation of the survey object);

- using TDI mode (CTDI $=4$, spline approximation of the survey object);

- without implementation of TDI mode (linear approximation of the survey object);

- using TDI mode (CTDI $=4$, linear approximation of the survey object).

\section{The results of modeling}

The results of satellite imagery modeling of lengthy territories with complex configuration using TDI mode are shown in figures $2 \ldots 4$ (angles in degrees, angular velocity in degrees/sec, time in sec).

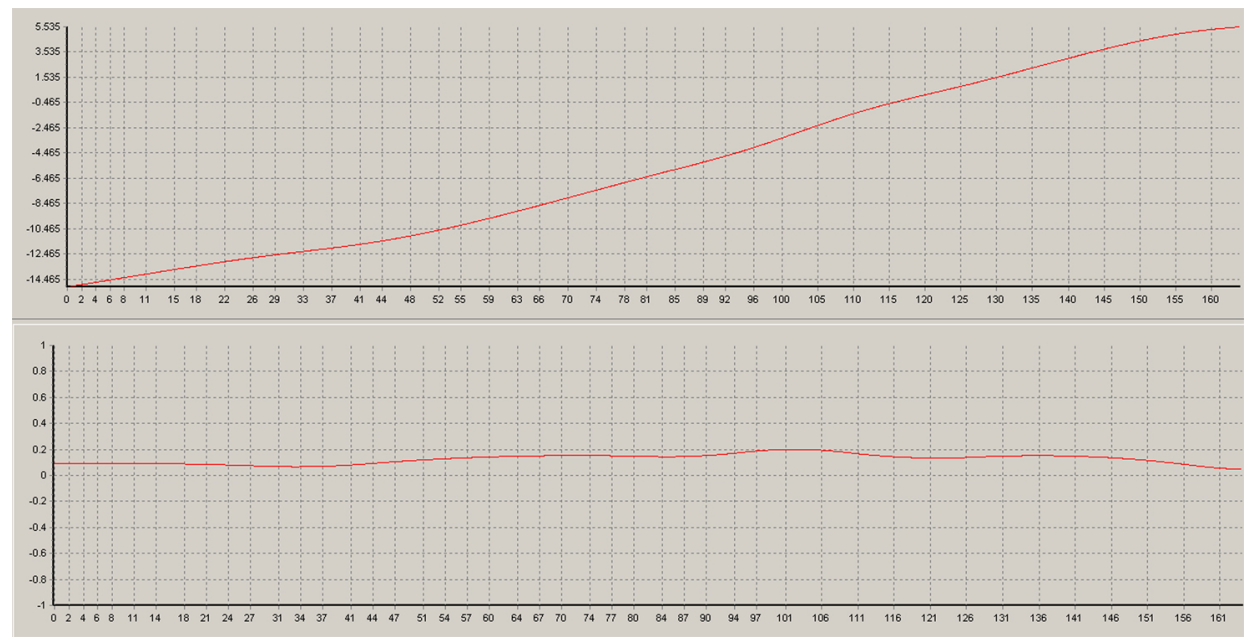

Fig. 2. Roll attitude angle (upper figure) and roll angular velocity (lower figure) during imagery using TDI mode (spline approximation of the survey object).

The results of modeling have shown that in one-orbit period it is possible to provide full coverage of the specified territory with spline approximation of the survey object, while linear approximation of the object being imaged allows to scan the required territory only partially, although at lower angular speeds. 


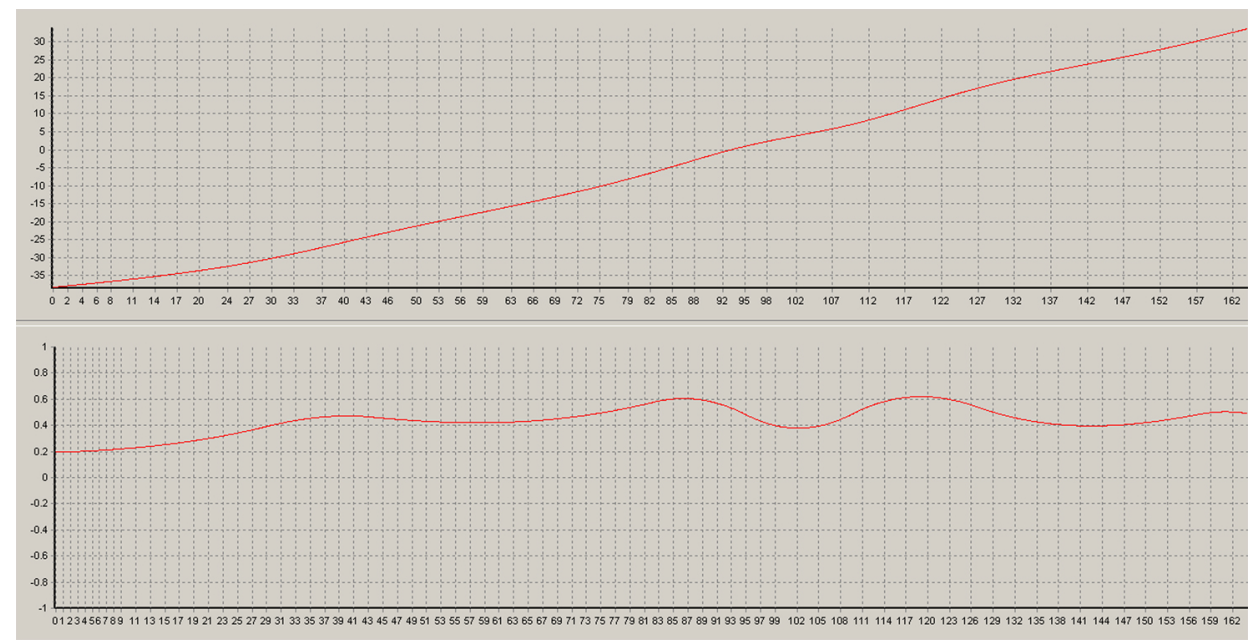

Fig. 3. Pitch angle (upper figure) and pitch angular velocity (lower figure) during imagery using TDI mode (spline approximation of the survey object).

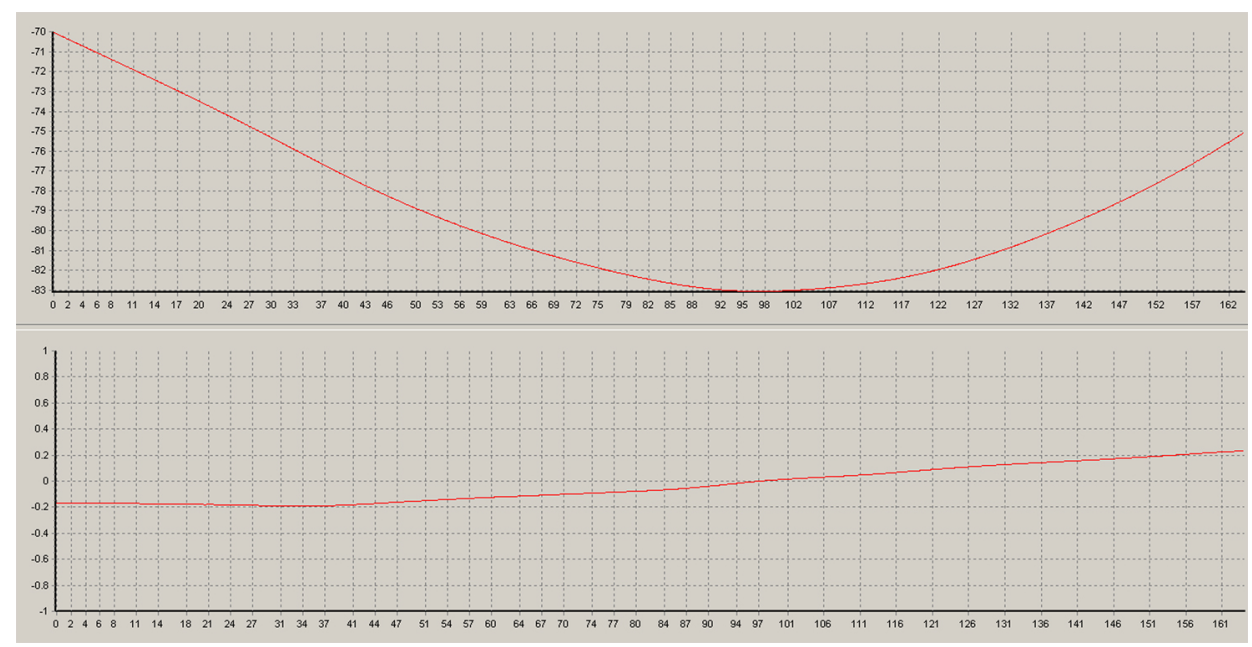

Fig. 4. Yaw angle (upper figure) and yaw angular velocity (lower figure) during imagery using TDI mode (spline approximation of the survey object).

Table 1 shows the ranges of attitude angles and satellite angular velocities required for various types of imagery, obtained as a result of modeling. 
Table 1. The ranges of attitude angles and satellite angular velocities

\begin{tabular}{|c|c|c|c|c|c|c|}
\hline \multirow[t]{2}{*}{$\begin{array}{l}\text { Approxi- } \\
\text { mation } \\
\text { type }\end{array}$} & \multirow{2}{*}{$\begin{array}{c}\text { Parameters } \\
\text { of attitude } \\
\text { and } \\
\text { satellite } \\
\text { angular } \\
\text { motion } \\
\end{array}$} & \multirow[t]{2}{*}{$\begin{array}{l}\text { Unit of } \\
\text { measu- } \\
\text { rement }\end{array}$} & \multicolumn{2}{|c|}{$\begin{array}{c}\text { Imagery without } \\
\text { implementation of TDI } \\
\text { mode (the duration of } \\
\text { imagery } 46 \mathrm{sec}) \\
\end{array}$} & \multicolumn{2}{|c|}{$\begin{array}{l}\text { Imagery using TDI } \\
\text { mode (the duration of } \\
\text { imagery } 164 \mathrm{sec})\end{array}$} \\
\hline & & & Min value & Max value & Min value & Max value \\
\hline \multirow{6}{*}{ 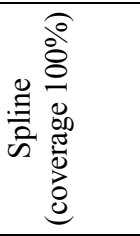 } & Roll angle & degree & -16.6 & 5.4 & -14.8 & 5.5 \\
\hline & Pitch angle & degree & -11.9 & 14.2 & -40 & 34 \\
\hline & Yaw angle & degree & -81 & -79.1 & -83 & -73 \\
\hline & Roll velocity & degree/sec & 0.29 & 0.65 & 0.08 & 0.21 \\
\hline & Pitch velocity & degree/sec & -0.05 & 1.4 & 0.2 & 0.62 \\
\hline & Yaw velocity & degree/sec & -0.21 & 0.39 & -0.2 & 0.23 \\
\hline \multirow{6}{*}{ 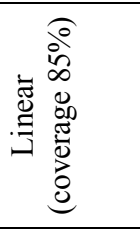 } & Roll angle & degree & -17.9 & 9 & -14.7 & 5.9 \\
\hline & Pitch angle & degree & -13.1 & 12.8 & -39 & 33 \\
\hline & Yaw angle & degree & -83.7 & -80.9 & -83.1 & -70.4 \\
\hline & Roll velocity & degree/sec & 0.45 & 0.52 & 0.05 & 0.18 \\
\hline & Pitch velocity & degree/sec & 0.51 & 0.58 & 0.29 & 0.52 \\
\hline & Yaw velocity & degree/sec & -0.22 & 0.09 & -0.2 & 0.21 \\
\hline
\end{tabular}

\section{Conclusion}

Computer modeling has confirmed the possibility of satellite imagery of lengthy territories with complex configuration at non-zero angular velocities using automatic satellite attitude program control system in the process of imagery. The angular velocities of satellite retargeting averaged less than $0.5 \mathrm{deg} / \mathrm{sec}$. The spline approximation of the survey object appeared to be the most effective type of approximation in terms of agility (in one-orbit period) and coverage percentage.

\section{References}

1. Z. Chen, J. Pasher, J. Duffe, A. Behnamian, Can. J. Remote Sens., 43, 513-527 (2017)

2. Y.-S. Hsiao, C. Hwang, Y.-S. Cheng, L.-C. Chen, H.-J. Hsu, J.-H. Tsai, C.-L. Liu, C.C. Wang, Y.-C. Liu, Y.-C. Kao, Remote Sens. Environ., 176, 69-83 (2016)

3. P.-C. Hsu, M.-H. Chang, C.-C. Lin, S.-J. Huang, C.-R. Ho, Remote Sens. Environ., 193, 54-64 (2017)

4. D.R. Rounce, C.S. Watson, D.C. McKinney, Remote Sens., 9, 654 (2017)

5. A. Shaker, W.Y. Yan, S. Easa, Geocarto. Int., 26, 49-67 (2011)

6. A.M. Youssef, B. Pradhan, N.H. Maerz, Arab. J. Geosci., 7, 2591-2601 (2014)

7. M. Amani, B. Salehi, S. Mahdavi, B. Brisco, ISPRS J. Photogramm., 144, 119-136 (2018)

8. Y. Duan, X. Huang, J. Xiong, Y. Zhang, B. Wang, Int. J. Digit. Earth, 9, 851-872 (2016)

9. M. Wang, Z. Zhang, Y. Zhu, Z. Dong, Y. Li, J. Real-Time Image Pr., 15, 565-581 (2018)

10. O.L. Makarov, D.K. Mozgovoy, V.S. Khoroshilov, 21th Int. Conf. on Microwave and Telecommunication Technology (CriMiCo), 905-907 (2011) 